\title{
Rh Positive Blood Group
}

National Cancer Institute

\section{Source}

National Cancer Institute. Rh Positive Blood Group. NCI Thesaurus. Code C76251.

A blood group indicating the presence on erythrocytes of the Rh D form of the Rhesus antigen. 